\title{
ChemComm
}

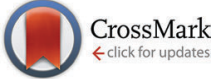

Cite this: Chem. Commun., 2016, 52, 2956

Received 10th July 2015, Accepted 12th January 2016

DOI: $10.1039 / c 5 c c 05743 d$

www.rsc.org/chemcomm

\section{A narrow amide I vibrational band observed by sum frequency generation spectroscopy reveals highly ordered structures of a biofilm protein at the air/water interface $\uparrow$}

\author{
Zhuguang Wang, ${ }^{a}$ M. Daniela Morales-Acosta, ${ }^{b}$ Shanghao Li, ${ }^{c}$ Wei Liu, ${ }^{a}$ \\ Tapan Kanai, ${ }^{a}$ Yuting Liu, ${ }^{a}$ Ya-Na Chen, ${ }^{a}$ Frederick J. Walker, ${ }^{b}$ Charles H. Ahn, ${ }^{b}$ \\ Roger M. Leblanc ${ }^{c}$ and Elsa C. Y. Yan*a
}

\begin{abstract}
We characterized BslA, a bacterial biofilm protein, at the air/water interface using vibrational sum frequency generation spectroscopy and observed one of the sharpest amide I bands ever reported. Combining methods of surface pressure measurements, thin film $X$-ray reflectivity, and atomic force microscopy, we showed extremely ordered BslA at the interface.
\end{abstract}

Biofilms are communities of microorganisms where cells are densely packed and attached to surfaces in extracellular matrices that consist mainly of exopolysaccharides, nucleic acids, and proteins. ${ }^{1}$ Biofilms constitute a protective mode of microbes to survive under hostile conditions. ${ }^{2}$ Biofilms facilitate gene transfer, quorum sensing, predation, and competition among colonies. ${ }^{3}$ Bacteria are shielded in biofilms from antimicrobial agents, making chronic infections a major challenge for the implantation of medical devices and bone replacement. ${ }^{2,4}$ Biofilms are characteristic of their amphiphilicity and stability, providing insights into molecular design of biomimetic materials, such as those used in biocontrol agents, inhibitors against metal corrosion, and bioreactors. ${ }^{5}$ Moreover, BslA is among the proteins that carry extreme properties, e.g., proteins expressed in thermophile with extremely high thermal stability, rhodopsin having extreme sensitivity for light detection for dim-light vision, antifreeze proteins lowering the freezing point to help animals survive at extremely low temperature, etc. Understanding the properties of these proteins not only is significant in protein science but also provides insight into molecular design of biomaterials. Since BslA is a newly identified protein shown to have extreme surface

\footnotetext{
${ }^{a}$ Department of Chemistry, Yale University, 225 Prospect Street, New Haven, Connecticut 06520, USA. E-mail: elsa.yan@yale.edu

${ }^{b}$ Department of Applied Physics, Yale University, 15 Prospect Street, New Haven, Connecticut 06520, USA

${ }^{c}$ Department of Chemistry, University of Miami, 1301 Memorial Drive,

Coral Gables, Florida 33146, USA

$\dagger$ Electronic supplementary information (ESI) available: Methods of BslA expression and purification; SFG setup; experimental procedure, data acquisition, and data analyses; supplementary data. See DOI: 10.1039/c5cc05743d
}

activity, characterization of its surface properties is therefore urgent and important.

Biofilms contain proteins as a major component. Some of these proteins are highly amphiphilic, belonging to the class of hydrophobins that facilitate formation of biofilms. Here, we focus on BslA (or YuaB) found in the biofilm of gram-positive bacteria, Bacillus subtilis that can grow on plants. ${ }^{6-8}$ BslA is known to facilitate the assembly of the extracellular matrix. ${ }^{6,7}$ Its crystal structure has been recently solved by Hobley et al. (Fig. 1A). ${ }^{9}$ The structure shows that 11 hydrophobic residues form a hydrophobic "cap" (green, Fig. 1A) and other residues form mainly hydrophilic $\beta$-strands (purple, Fig. 1A), exhibiting strong amphiphilicity. ${ }^{9}$ BslA shares little sequence homology and structural similarity with other hydrophobins. ${ }^{10}$ Uniquely, BslA does not use disulphide-bonded networks to stabilize surface structures. Instead, as Bromley et al. proposed,$^{10}$ it changes conformations in the "cap" region from disordered loops to $\beta$-sheets (green, Fig. 1A) upon surface adsorption, exposing the hydrophobic residues for stabilization at interfaces. ${ }^{10}$ However, the molecular packing, structure, and orientation of BslA at the interfaces have not been fully understood, which require surface-specific methods for comprehensive characterizations.

Here, we combined surface-specific vibrational sum frequency generation spectroscopy (SFG) with surface pressure measurements, atomic force microscopy, and thin-film X-ray reflectivity to characterize BslA at the air/water interface, which is a model system for hydrophobic/hydrophilic interfaces. We expressed and purified a truncated version of BsIA (see ESI $\dagger$ for procedures) with amino acids $29-176$, the same construct as the one in the crystal structure. ${ }^{9}$ This truncated version was shown to be fully functional with higher stability. ${ }^{7,9}$ Using SFG, we observed an unusually narrow amide I vibrational band of the protein backbone from BslA at the air/water interface. Since SFG is very sensitive to molecular ordering and orientation at interfaces, the result prompted us to hypothesize that BslA forms an extremely ordered and neatly oriented structure at the air/water interface. Below, we report the observation of this narrow amide I band and the experiments to test this hypothesis. 

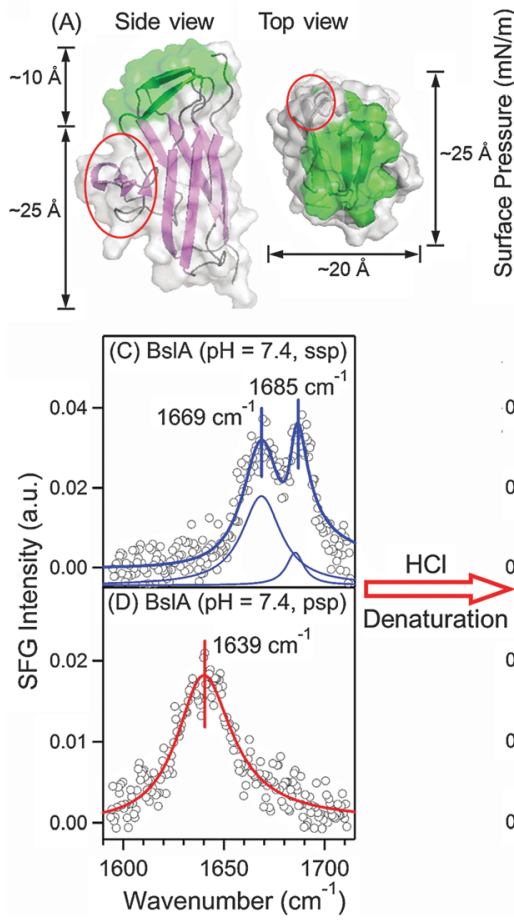

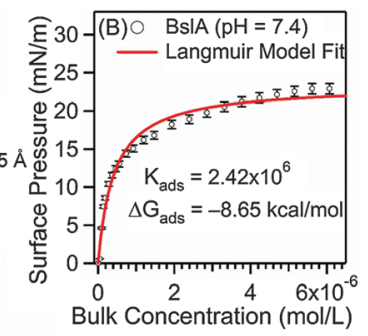

(E) $\mathrm{Bs} / \mathrm{A}(\mathrm{pH}=1.2, \mathrm{ssp})$

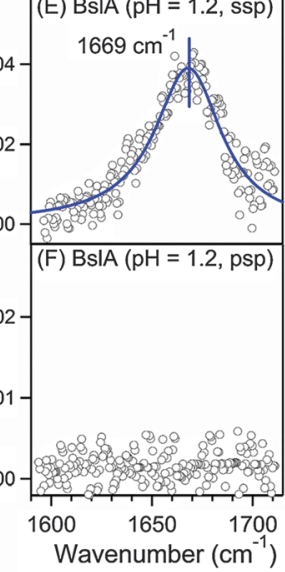

Fig. 1 Surface characterization of BslA at the air/water interface. (A) Crystal structure of BslA: ${ }^{9}$ the hydrophobic (green) and hydrophilic domains (purple), and the protruding region (red). (B) Surface adsorption isotherm of BslA at the air/water interface, ( $n=3, \pm$ S.D. as error bars). (C) Achiral (ssp) and (D) chiral (psp) SFG spectra of BslA at the air/water interface at $\mathrm{pH} 7.4$ with a concentration of $6 \mu \mathrm{M}$. (E) Achiral (ssp) and (F) chiral (psp) SFG spectra of BsIA at $\mathrm{pH} 1.2$ with a bulk concentration of $6 \mu \mathrm{M}$. For $(C)-(E)$, the spectral fitting parameters are provided in Table 1.

We first studied the adsorption and self-assembly process of BslA at the air/water interface. We obtained the adsorption isotherm at $\mathrm{pH} 7.4$ (buffer: $10 \mathrm{mM}$ phosphate and $100 \mathrm{mM}$ $\mathrm{NaCl}$ ) and $23{ }^{\circ} \mathrm{C}$ by monitoring the surface pressure at increasing concentrations of BslA. Fig. 1B shows that the surface pressure increases drastically at bulk concentrations lower than $1 \mu \mathrm{M}$, indicating strong adsorption of BslA at the interface. When the bulk concentration exceeds $1 \mu \mathrm{M}$, the interface is gradually saturated with BslA until reaching a maximum surface pressure of $\sim 23 \mathrm{mN} \mathrm{m}^{-1}$ at $\sim 6 \mu \mathrm{M}$. The adsorption isotherm is fitted with the Langmuir model (see ESI $\dagger)^{11}$ as the red curve in Fig. 1B. The fitting yields an adsorption free energy $\left(\Delta G^{\circ}\right)$ of $-8.65 \mathrm{kcal} \mathrm{mol}^{-1}$. This large negative $\Delta G^{\circ}$ reveals the strong surface activity of BslA at the air/water interface, even stronger than the amphiphilic longchain alcohol, $\mathrm{CH}_{3}\left(\mathrm{CH}_{2}\right)_{9} \mathrm{OH}$, with $\Delta G^{\circ}$ of $-6.64 \mathrm{kcal} \mathrm{mol}^{-1} \cdot{ }^{12}$

We then used vibrational SFG spectroscopy to characterize BslA at the air/water interface at the saturated level with a bulk concentration of $6 \mu \mathrm{M}$. Vibrational SFG spectroscopy is a non-invasive, label-free, and surface-selective vibrational spectroscopic method, ${ }^{13}$ which has been used to study biomolecules at interfaces. ${ }^{14,15}$ Since amide I bands are characteristic of protein secondary structures, ${ }^{16}$ we first acquired the conventional (achiral) SFG spectrum of BslA in the amide I vibrational region (1600-1700 $\left.\mathrm{cm}^{-1}\right)$ using the ssp polarization setting (s-polarized SFG, s-polarized visible, and p-polarized infrared) (setup shown in ESI $\dagger$ ).
Table 1 Fitting parameters of achiral and chiral SFG spectra for BsIA

\begin{tabular}{lcc}
\hline Parameters & Amide I (achiral-ssp) Fig. 1C (pH 7.4) \\
\hline$\chi_{\mathrm{NR}}($ a.u.) & $0.020 \pm 0.005$ & \\
$\omega\left(\mathrm{cm}^{-1}\right)$ & $1668.5 \pm 0.7$ & $1685.3 \pm 0.4$ \\
$A($ a.u) & $1.99 \pm 0.24$ & $0.53 \pm 0.16$ \\
$\Gamma$ (a.u.) & $11.75 \pm 1.29$ & $5.25 \pm 1.09$ \\
\hline & Amide I (chiral-psp) & Amide I (achiral-ssp) \\
Parameters & Fig. $1 \mathrm{D}(\mathrm{pH} 7.4)$ & Fig. $1 \mathrm{E}(\mathrm{pH} 1.2)$ \\
\hline$\chi_{\mathrm{NR}}($ a.u.) & $0.008 \pm 0.003$ & $-0.005 \pm 0.003$ \\
$\omega$ (cm $\left.{ }^{-1}\right)$ & $1639.1 \pm 0.9$ & $1668.6 \pm 0.8$ \\
$A$ (a.u.) & $2.23 \pm 0.07$ & $3.97 \pm 0.10$ \\
$\Gamma$ (a.u.) & $16.65 \pm 0.69$ & $20.15 \pm 0.62$ \\
& &
\end{tabular}

The amide I spectrum (Fig. 1C) gives surprisingly sharp spectral features. Fitting the spectrum (Table 1), we identified two vibrational bands and assigned them to $\beta$-turn $\left(1669 \mathrm{~cm}^{-1}\right)$ and the $B_{1}$ mode of antiparallel $\beta$-sheets $\left(1685 \mathrm{~cm}^{-1}\right)$. These assignments are consistent with the standard amide I frequencies for the secondary structures ${ }^{16}$ and the crystal structure of BslA, with extensive antiparallel $\beta$-sheet strands connected by $\beta$-turns (Fig. 1A). ${ }^{9}$ Notably, the damping coefficient for the $1685 \mathrm{~cm}^{-1}$ band is only $5.25 \mathrm{~cm}^{-1}$, corresponding to a full-width-at-halfmaximum (FWHM) of $10.50 \mathrm{~cm}^{-1}$, approaching the spectral resolution of our broad bandwidth SFG spectrometer $\left(\sim 8 \mathrm{~cm}^{-1}\right)$. This amide I band is broader at lower BslA bulk concentration (Table S2, ESI $\dagger$ ). It is much narrower than that for common proteins, which typically have FWHM greater than $20 \mathrm{~cm}^{-1}$ for single secondary structures observed using conventional vibrational methods, such as infrared and SFG (Table S3, ESI $\dagger$ ). ${ }^{16,17}$ Our observed sharp amide I band is unusual because inhomogeneity of the amide groups in protein structures and various extents of hydrogen-bonding interactions with solvents often broaden the amide I band. Since SFG signals depend highly on molecular ordering and orientation, the sharp amide I band (Fig. 1C) led to the hypothesis that BslA self-assembles into extremely ordered and compact structures at the air/water interface.

We further acquired the chiral SFG spectrum using the psp polarization setting ( $\mathrm{p}$-polarized SFG, s-polarized visible, and p-polarized IR) under the same conditions (Fig. 1D). Chiral SFG has the advantages of being free of spectral background from achiral molecules, including water. ${ }^{18-20}$ Due to the difference in selection rules, ${ }^{20}$ the chiral SFG spectrum (Fig. 1D) of BslA at the air/water interface is different from the achiral SFG spectrum (Fig. 1C). It exhibits only one amide I band at $1639 \mathrm{~cm}^{-1}$, which is at the high-frequency end of the antiparallel $\beta$-sheet $B_{2}$ vibrational mode and the low-frequency end of disordered structures. ${ }^{16} \mathrm{We}$ ruled out the assignment of disordered structures because they should not exhibit chiral amide I SFG signals. ${ }^{18,20}$ Thus, we assigned the $1639 \mathrm{~cm}^{-1}$ band to the $B_{2}$ vibrational mode of antiparallel $\beta$-sheets, consistent with the assignment of the $1685 \mathrm{~cm}^{-1}$ band in the achiral spectrum (Fig. 1C) to the $B_{1}$ mode of antiparallel $\beta$-sheets. ${ }^{16}$

We then examined the narrow $1685 \mathrm{~cm}^{-1}$ band (Fig. 1C) under acid-denaturing conditions. We added $\mathrm{HCl}$ solution to decrease the $\mathrm{pH}$ from 7.4 to 1.2 to denature BslA, and then obtained the achiral and chiral SFG spectra. The achiral SFG 
spectrum shows that the sharp band at $1685 \mathrm{~cm}^{-1}$ vanishes, leaving a broad band at $1669 \mathrm{~cm}^{-1}$ (Fig. 1E), while the chiral SFG spectrum shows that the $1639 \mathrm{~cm}^{-1}$ band disappears (Fig. 1F). The vanishing vibrational bands at $1685 \mathrm{~cm}^{-1}$ in the achiral spectrum (Fig. 1C) and $1639 \mathrm{~cm}^{-1}$ in the chiral spectrum (Fig. 1D) indicate that the acidic condition denatures the antiparallel $\beta$-sheets at the interface. The remaining band at $1669 \mathrm{~cm}^{-1}$ in the achiral spectrum (Fig. 1E) can originate from $\beta$-turns ${ }^{16}$ and/or disordered structures commonly found in denatured proteins. ${ }^{21}$ In addition, the damping coefficient of the $1669 \mathrm{~cm}^{-1}$ band in the achiral spectra increases from $11.75 \mathrm{~cm}^{-1}$ (Fig. 1C) to $16.65 \mathrm{~cm}^{-1}$ (Fig. 1E), suggesting that the denatured protein is more disordered and/or more inhomogeneous. The disappearance of the achiral $1685 \mathrm{~cm}^{-1}$ narrow band and the chiral $1639 \mathrm{~cm}^{-1}$ band is due to the structural changes, rather than desorption of BslA from the interface as the intensity of the achiral amide I band $\left(1669 \mathrm{~cm}^{-1}\right)$ is comparable to that before denaturation. These results show that the unusually narrow amide I vibrational band at the neutral pH (Fig. 1C) comes from properly assembled, folded BslA at the air/water interface.

To further evaluate the surface activity of BslA, we obtained its surface compression isotherm. We monitored the surface pressure while compressing the BslA monolayer at the air/water interface using two barriers in a Langmuir trough (Fig. 2A), a common method used for studying stability and packing of monolayers of amphiphilic molecules, e.g. detergent and lipid, at the air/water interface (see ESI $\dagger$ ). We first observed a rise of surface pressure at $\sim 1300 \AA^{2}$ per molecule and then a clear phase transition from the liquid to the solid phases at the surface pressure of $5-10 \mathrm{mN} \mathrm{m}^{-1}$, followed by a steep increase of surface pressure, similar to the compression isotherms of the monolayer of highly rigid saturated lipids, such as DPPG. ${ }^{15}$ In principle, a clear phase transition in surface compression isotherm indicates high rigidity and ordered packing of monolayers that are typical for small amphiphilic molecules but uncommon for macroscopic molecules. Thus, as a protein, BslA shows extraordinary amphiphilicity with exceptional self-assembling ability. The BslA monolayer collapses at $\sim 45 \mathrm{mN} \mathrm{m}^{-1}$, where the surface pressure stops rising upon compression. This collapsing surface pressure is higher than the typical value for macroscopic proteins in the range of $25-35 \mathrm{mN} \mathrm{m}^{-1}$ but comparable to that for lipid molecules. $^{22}$ This result suggests that the monolayer of BslA is capable of forming stable membrane-like structures, similar to the ones formed by phospholipid. By fitting the linear portion of
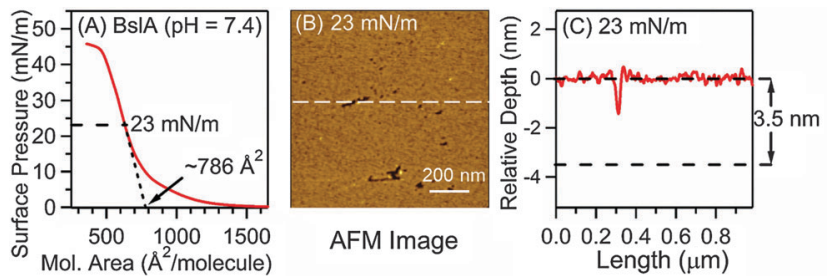

Fig. 2 (A) Surface compression isotherm of BsIA at the air/water interface at $\mathrm{pH}$ 7.4. (B) Representative AFM image of BslA monolayer on mica prepared at the surface pressure of $23 \mathrm{mN} \mathrm{m}^{-1}$ at the air/water interface, with the corresponding depth profile at the white dashed line presented in (C). AFM images probed in different probing areas are presented in the ESI. $\dagger$ the surface isotherm and extending the fitted line (see ESI $\dagger$ ) to $0 \mathrm{mN} \mathrm{m}^{-1}$, we obtained the limiting molecular area as $786 \pm 8 \AA^{2}$ per molecule, which is $\sim 60 \%$ larger than the cross sectional area $\left(\sim 500 \AA^{2}\right)$ of the crystal structure oriented with the hydrophobic "cap" pointing up (top view, Fig. 1A). The ratio of the limiting area to the cross sectional area of BslA is significantly smaller than that of common proteins, such as insulin that has a limiting area 500\% larger than the cross sectional area of its crystal structure. ${ }^{23}$ Altogether, the surface compression isotherm supports that BslA can form a robust, tightly packed, and rigid membranelike structure at the air/water interface.

To evaluate the packing of the BslA monolayer, we then used atomic force microscopy (AFM) to characterize Langmuir-Blodgett (LB) films of BslA. We made the LB film by vertically pulling a piece of mica from the solution in the Langmuir trough containing a monolayer of BslA at a constant speed while maintaining the surface pressure of $23 \mathrm{mN} \mathrm{m}^{-1}$ (see $\mathrm{ESI} \dagger$ ), the saturated surface pressure in the adsorption isotherm (Fig. 1B). Fig. 2B shows the representative AFM image of the LB films, revealing the morphology of BslA at the interface (see also other images in Fig. S3, ESI $\dagger$ ). It shows a smooth coverage of BslA molecules with only a few minor defects over a macroscopic length of $1 \mu \mathrm{m}$. Fig. 2C shows the corresponding depth profile, indicating that the defects are $\sim 1.4 \mathrm{~nm}$ deep. With the assumption that BslA vertically orients at the mica surface with the hydrophobic "cap" facing up, two lines are drawn to depict the approximate height of BslA $(3.5 \mathrm{~nm})$ shown in the crystal structure (Fig. 1A). ${ }^{9}$ We proposed that the defects are likely due to BslA molecules lying down on the mica surface and the large smooth coverage in the macroscopic length scale of $\mu \mathrm{m}$ is due to densely packed, highly ordered BslA molecules with the cap facing up. We further examined this proposed molecular picture using thin film X-ray reflectivity.

We performed thin film X-ray reflectivity (XRR) experiments to further characterize the LB film of BslA prepared at the same surface pressure of $23 \mathrm{mN} \mathrm{m}^{-1}$. The log reflectivity curve (Fig. 3A) exhibits a clear fringe pattern as a result of the interference between the partially reflected X-rays from the air/film and film/ substrate interfaces. We also observed peaks associated with Bragg reflections (peaks at $\sim 4.5^{\circ}$ and $\sim 9.0^{\circ}$ ), which are characteristic of the mica crystalline substrate. The XRR result for mica without the LB film shows the same Bragg reflection peaks without a fringe pattern (Fig. S4, ESI $\dagger$ ). We analyzed the XRR pattern for the BslA film, and generated a three-layered fitting model (solid red line, Fig. 3A and Table S1, ESI $\dagger$ ): (1) a $9.5 \AA$ thick hydrophobic cap with a density of $1.25 \mathrm{~g} \mathrm{~cm}^{-3}$, (2) a $22.0 \AA$ thick BslA body with a density of $1.14 \mathrm{~g} \mathrm{~cm}^{-3}$, and (3) a 4.2 A thick tail with a density of $0.18 \mathrm{~g} \mathrm{~cm}^{-3}$. The fitting gives an occupied area of $610 \pm 20 \AA^{2}$ per molecule. This is larger than the lateral dimensions of the crystal structure, i.e. $\sim 500 \AA^{2}$ (Fig. 1A), but is consistent with the molecular area $\left(\sim 630 \AA^{2}\right)$ at $23 \mathrm{mN} \mathrm{m}^{-1}$ shown in the compression isotherm (Fig. 2A). We speculate that the difference between the occupied area and the cross-sectional area of the crystal structure can result from the conformational changes in the protruding region (Fig. 1A, circled in red). This region contains a segment of 310 -helix, a relatively unstable secondary structure compared with $\alpha$-helices and $\beta$-sheets. In the crystal structure, the average 

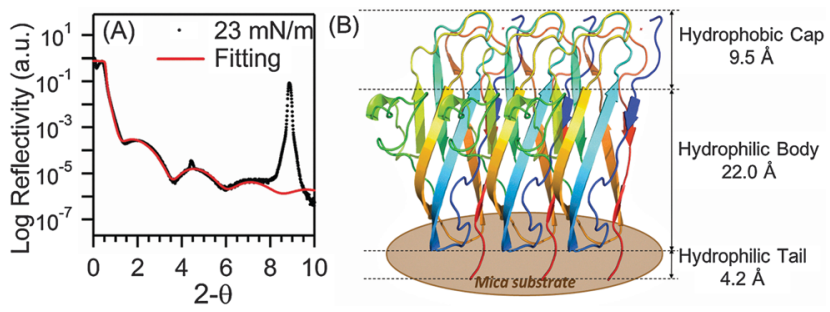

Fig. 3 (A) X-ray reflectivity curve of BslA monolayer on mica prepared at the surface pressure of $23 \mathrm{mN} \mathrm{m}^{-1}$. Fitting parameters for the fitted line (red) are provided in ESI. $\dagger$ (B) The three-layered model of BsIA monolayer on mica, derived from XRR analysis.

B-factor in this region (33) is higher than that in the regions of $\beta$-sheets (25), indicating higher flexibility. In vivo studies showed that bacteria expressing BslA with mutations in this region do not exhibit significant changes in hydrophobicity of the biofilms. ${ }^{10}$ However, we cannot exclude the possibility that this region may still undergo conformational changes to facilitate intermolecular interactions. The total thickness and values of the density in the fitting model are consistent with the crystal structure (Fig. 1A) at a full surface coverage that orients vertically with the higher density hydrophobic cap facing up (Fig. 3B), confirming the formation of a densely packed monolayer of BslA at the air/water interface.

In summary, we observed an unusually narrow amide I vibrational band in the SFG spectrum of BslA at the air/water interface. We further characterized BslA at the air/water interface by performing surface pressure measurements, AFM imaging, and thin film X-ray reflectivity experiments. On the basis of these characterization, we attributed the narrow amide I band to the extremely ordered, neatly oriented, and highly compact structures of BslA at the interface, consistent with the recent report of highly ordered 2D lattices formed by BslA. ${ }^{10}$ Our results also support that BslA can likely form a stable and robust outmost layer at the air/ water or hydrophobic/water interfaces as a protective shield for microbial growth in biofilms.

Our study of surface properties of BslA at the air/water interface has some implications. First, the surprisingly sharp amide I vibrational band observed by SFG can be used as a sensitive in situ and real-time tool to examine the highly ordered structures of BslA at the air/water interfaces. In conjunction with mutagenesis, this vibrational band will provide highly informative readout to reveal the molecular mechanism of the exceptional surface activity of BslA. Second, our study shows the strong surface activity, tight packing, and neatly ordered structure of BslA at the air/water interface, providing a basis for the design and synthesis of new surface-active biomimetic materials. Finally, our work reinforces the proposal that BslA likely forms the outermost surface structures of biofilms to protect microbial growth. ${ }^{10}$ Further structure-function studies of BslA as a model system of biofilm proteins can potentially introduce strategies for perturbing biofilm structures to enhance the efficacy of antibiotics for treating infectious diseases.

This work is supported by the National Science Foundation (NSF) Grant CHE 1213362 and National Institutes of Health
(NIH) Grant 1R56DK105381-01 to E. C. Y. Y., CRISP MRSEC NSF DMR 1119826 to C. H. A., and CBET 1355317 to R. M. L. M. D. M. A. is partially supported by a Postdoctoral Fellowship of Mexico's National Council of Science and Technology.

\section{Notes and references}

1 J. W. Costerton, Z. Lewandowski, D. E. Caldwell, D. R. Korber and H. M. Lappinscott, Annu. Rev. Microbiol., 1995, 49, 711-745; M. E. Davey and G. A. O’toole, Microbiol. Mol. Biol. Rev., 2000, 64, 847-867; H. C. Flemming and J. Wingender, Nat. Rev. Microbiol., 2010, 8, 623-633.

2 J. W. Costerton, P. S. Stewart and E. P. Greenberg, Science, 1999, 284, 1318-1322.

3 R. M. Donlan, Emerging Infect. Dis., 2002, 8, 881-890.

4 L. Hall-Stoodley, J. W. Costerton and P. Stoodley, Nat. Rev. Microbiol., 2004, 2, 95-108; P. S. Stewart and J. W. Costerton, Lancet, 2001, 358, 135-138.

5 M. Morikawa, J. Biosci. Bioeng., 2006, 101, 1-8; G. Seneviratne, R. M. M. S. Thilakaratne, A. P. D. A. Jayasekara, K. A. C. N. Seneviratne, K. R. E. Padmathilake, M. S. D. L. De Silva, Microbial Strategies for Crop Improvement, 2009, vol. 51, p. 62.

6 K. Kobayashi and M. Iwano, Mol. Microbiol., 2012, 85, 51-66; S. S. Branda, F. Chu, D. B. Kearns, R. Losick and R. Kolter, Mol. Microbiol., 2006, 59, 1229-1238; D. B. Kearns, F. Chu, S. S. Branda, R. Kolter and R. Losick, Mol. Microbiol., 2005, 55, 739-749.

7 A. Ostrowski, A. Mehert, A. Prescott, T. B. Kiley and N. R. StanleyWall, J. Bacteriol., 2011, 193, 4821-4831.

8 A. T. Kovacs, J. van Gestel and O. P. Kuipers, Mol. Microbiol., 2012, 85, 8-11.

9 L. Hobley, A. Ostrowski, F. V. Rao, K. M. Bromley, M. Porter, A. R. Prescott, C. E. MacPhee, D. M. F. Van Aalten and N. R. Stanley-Wall, Proc. Natl. Acad. Sci. U. S. A., 2013, 110, 13600-13605.

10 K. M. Bromley, R. J. Morris, L. Hobley, G. Brandani, R. M. C. Gillespie, M. McCluskey, U. Zachariae, D. Marenduzzo, N. R. StanleyWall and C. E. MacPhee, Proc. Natl. Acad. Sci. U. S. A., 2015, 112, 5419-5424.

11 I. Langmuir, J. Am. Chem. Soc., 1918, 40, 1361-1403.

12 M. J. Rosen and S. Aronson, Colloids Surf., 1981, 3, 201-208.

13 Y. Shen, Nature, 1989, 337, 519-525; K. B. Eisenthal, Chem. Rev., 1996, 96, 1343-1360; G. L. Richmond, Chem. Rev., 2002, 102, 2693-2724; H. F. Wang, W. Gan, R. Lu, Y. Rao and B. H. Wu, Int. Rev. Phys. Chem., 2005, 24, 191-256; A. G. Lambert, P. B. Davies and D. J. Neivandt, Appl. Spectrosc. Rev., 2005, 40, 103-145.

14 G. L. Richmond, Anal. Chem., 1997, 69, A536-A543; T. Weidner, J. S. Apte, L. J. Gamble and D. G. Castner, Langmuir, 2010, 26, 3433-3440; J. Wang, C. Y. Chen, S. M. Buck and Z. Chen, J. Phys. Chem. B, 2001, 105, 12118-12125; J. Kim and G. A. Somorjai, J. Am. Chem. Soc., 2003, 125, 3150-3158; M. F. M. Engel, C. C. VandenAkker, M. Schleeger, K. P. Velikov, G. H. Koenderink and M. Bonn, J. Am. Chem. Soc., 2012, 134, 14781-14788.

15 W. Liu, Z. G. Wang, L. Fu, R. M. Leblanc and E. C. Y. Yan, Langmuir, 2013, 29, 15022-15031.

16 A. Barth and C. Zscherp, Q. Rev. Biophys., 2002, 35, 369-430; L. K. Tamm and S. A. Tatulian, Q. Rev. Biophys., 1997, 30, 365-429.

17 E. Goormaghtigh, V. Cabiaux and J. M. Ruysschaert, Subcell. Biochem., 1994, 23, 405-450; Y. W. Liu, J. Jasensky and Z. Chen, Langmuir, 2012, 28, 2113-2121.

18 L. Fu, J. Liu and E. C. Y. Yan, J. Am. Chem. Soc., 2011, 133, 8094-8097; L. Fu, Z. G. Wang and E. C. Y. Yan, Int. J. Mol. Sci., 2011, 12, 9404-9425; E. C. Y. Yan, L. Fu, Z. G. Wang and W. Liu, Chem. Rev., 2014, 114, 8471-8498.

19 L. Fu, D. Q. Xiao, Z. G. Wang, V. S. Batista and E. C. Y. Yan, J. Am. Chem. Soc., 2013, 135, 3592-3598; Z. Wang, L. Fu and E. C. Y. Yan, Langmuir, 2013, 29, 4077-4083.

20 E. C. Y. Yan, Z. Wang and L. Fu, J. Phys. Chem. B, 2015, 119, 2769-2785.

21 J. Wang, X. Y. Chen, M. L. Clarke and Z. Chen, Proc. Natl. Acad. Sci. U. S. A., 2005, 102, 4978-4983.

22 I. Langmuir and V. J. Schaefer, Chem. Rev., 1939, 24, 181-202.

23 S. Johnson, W. Liu, G. Thakur, A. Dadlani, R. Patel, J. Orbulescu, J. D. Whyte, M. Micic and R. M. Leblanc, J. Phys. Chem. B, 2012, 116, 10205-10212. 